\title{
Understanding the Adoption Intention of AI through the Ethics Lens
}

\author{
Ohbyung Kwon \\ School of Management \\ Kyung Hee University \\ obkwon@khu.ac.kr
}

\author{
Sujin Bae \\ School of Management \\ Kyung Hee University \\ luckybsj@khu.ac.kr
}

\author{
Bongsik Shin \\ Fowler College of Business \\ San Diego State University \\ bshin@sdsu.edu
}

\begin{abstract}
Understanding users and user behaviors in accepting new technologies such as AI has been ever more important. Meanwhile, information systems with AI inevitably engenders such ethical issues as transparency and accountability related to the consequences of recognition, decisions, and recommendations. Our work adds moral psychology variables to Theory of Reasoned Action (TRA) in order to better explicate the adoption aspects of AI. For the research, we employed social desirability, and selfconsistency of moral psychology as underlying attitudes. And also, moral norm is added to TRA to moderate the effect of the attitudes on the outcome variable. The empirical results indicate a direct and indirect role of the morality-related variables in explaining users' AI adoption intentions. It was learned that moral psychology plays an important role in explaining user attitudes toward AI and subsequent intentions of adopting an AI system.
\end{abstract}

\section{Introduction}

Artificial Intelligence (AI) refers to a system that can sense, comprehend, act and learn. AI technology has been rapidly rising with two primary motivations: (1) transfer human's intelligence and information processing capability to the machine, mimicking human's information-processing and cognitive psychology; and (2) effective handling of machine/system tasks powered by human-like cognitive capacity. AI of the second type is intended to support individual- or business performance by embedding AI within an information system. Among the numerous platforms and applications that are increasingly powered by AI are knowledge management [38], stock market prediction [39], intelligent tutoring system [61], intelligent manufacturing [47], big data analytics, deep learning, and Internet of Things [32]. There are also many other products such as self-driving vehicles, drones, and medical equipment that rely on AI for their enhanced performance. Coupled with the AI's meteoric rise, comprehending the dynamism of people's reactions to $\mathrm{AI}$ is becoming important for businesses to better respond to consumers and predict their behaviors.

Meanwhile, various technology acceptance theories and models have been introduced to explain the adoption decision and behaviors of new innovations such as AI. Among them are: Theory of Reasoned Action (TRA) [4], Theory of Planned Behavior [2], Technology Acceptance Model [18] [19] and Extended TAM [57], Unified Theory of Acceptance and Use of Technology (UTAUT)[58], Motivational Model [20], the model combining TAM and Theory of Planned Behavior [52], Innovation Diffusion Theory [43] and Social Cognitive Theory [10].

The traditional technology adoption/usage theories, however, may not be enough to fully explain human behaviors in facing AI. AI can self-judge and control own behaviors and thus distinctively different from those of traditional information systems with no such intelligence capabilities. Especially, the effects of an AI system on individuals, groups, and the society can be highly detrimental. AI, thus, raises grave implications on humans, making it fundamentally different from conventional information systems.

Besides, AI inevitably engenders serious ethical issues as opportunities for their abuse are abound [28]. Among them are privacy breaches, abuse of genetic data banking, digital divide, intercultural information ethics, and the use of social media [28]. The Google engine's misclassification of a person's photo as a gorilla and Microsoft chatbot's racist tweets reflect flawed training and abuse of AI by humans although these were unintended. In fact, much of the existing problem is not AI itself but rests on humans in embracing the technology [62].

With the growing ethical issues of AI, there have been attempts to develop a framework intended to guide ethical decision-making by AI [17]. Google and Microsoft already embraced an AI Ethics Board/Council and IEEE has issued recommendations 
on the ethical usage of AI. They underscore the criticality of factoring in ethical elements to explain the adoption/usage of AI. Despite, existing theoretical models (e.g., TAM) that have been embraced by IS scholars largely explain the adoption decision of IT primarily through the utilitarian and hedonic lens. Although there have been attempts to add such ethical factors as personal norm or moral norm to current theories such as TRA [56], little research investigated their role in the context of AI. Further, no studies have conducted their dynamism in the presence of other ethical elements (e.g., ethics recognition and attitudes) and this warrants an extended research model for AI.

Meanwhile, since its initial advancement by Rubin (1993)[45] and Ruggiero (2000)[44], uses and gratifications theory has garnered significant and increasing attention from technology scholars interested in user's active willingness and initiative to value emergent IT-based products such as media. For example, uses and gratification theory (U\&G) indicated that the consumers have already accepted the specific media to use and actively choose the media in order to fulfill their gratifications as well. This is well applied in understanding consuming new IT-based products [44]. Uses and gratifications theory originally focuses on why and what audiences do with mass communication tools [31] [51]. The positive willingness would contain ethical decision making, which takes ethical issues in account before active consumption. However, uses and gratifications theory and even uses and gratifications 2.0 [50] have not been applied in AI product context. Moreover, uses and gratifications theory can be extended its implications when adopting user's psychological mechanism on ethical reasoning. Thus, we need to extend uses and gratifications theory by connecting it with moral psychology, especially in the context of new AI product consumption.

Hence, we introduce an extended TRA research model, which includes the moral norm of AI as an internalized value of important others toward AI, to examine if it improves the TRA's explanatory power. Then, two variables of moral psychology are posited to shape individual attitudes toward AI (i.e., usefulness perception, subjective norm, and moral norm). The expanded research model, then, is empirically tested in its integrity. Distinctive patterns emerged from the empirical data analysis, highlighting the theoretical and practical importance of embracing ethical factors to better explicate AI adoption behaviors by users. Note that we argue that how the 'user's moral reasoning capability and user's attitude on the general ethical dilemma of AI', not just 'user's perception and attitude on the specific problem of a specific AI product' affect the intention to use new AI.

\section{Literature Review \& Theory}

More studies are being conducted by IS researchers with regards to adoption aspects of the AI system. Most of the studies on AI adoption is based on such adoption theories as TAM (Technology Acceptance Model) and UTAUT (Unified Theory of Acceptance and Use of Technology) and then expand them by adding such factors as hedonic value, trust, and technology diffusion. For example, Fan et al. (2018)[21] conducts the adoption of AI-based medical diagnosis through the combination of UTAUT and trust theory. Yang and Lee (2018)[63] attempts to explain the adoption of virtual personal assistant devices through perceived usefulness and perceived enjoyment. Akinnuwesi et al (2016)[5] explains biometric technology adoption through UTAUT. Efforts to explain a person's AI adoption behaviors through the lens of utilitarian and/or hedonic values appear in other AI studies as well. The adoption behaviors of smartwatches are explained based on the TAM's expanded model that contained hedonic motivation, a form of non-utilitarian motivation [16]. There are also studies that considered trust as an important antecedent of AI adoption [64] [21]. Hlee et al (2017)[27] approached AI adoption through the lens of diffusion of innovation on the ground that AI is a new innovation.

Our research is anchored on Theory of Reasoned Action (TRA) (see Figure 1), a case of the Theory of Planned Behavior (TPB), and extends it to investigate implications of ethics variables in explaining personal AI adoption behaviors. Compared to TAM (Technology Acceptance Model), TRA is more appropriate for our research as it contains subjective norm, a form of normative value. Figure 1 summarizes TRA's key constructs and their relationships in which the intention is a function of behavioral attitudes. Meanwhile, the attitude is the results of two belief considerations: behavioral belief as an individual's belief about certain consequences of a behavior (e.g., taking exercise will reduce my risk of heart disease) and outcome evaluations as positive or negative assessment of the behavior.

Meanwhile, subjective norm represents beliefs about whether key people approve or disapprove of a behavior and the motivation to behave in a way that gains their approval. It is, therefore, the level of perceived social pressures on an individual to engage or not engage in said behavior(s) [2]. The subjective norm is expected to be a function of normative beliefs and motivation to comply. Normative beliefs are an individual's beliefs about the extent other people who are important to him/her think he/she should or should not perform a particular behavior. Motivation to comply is about how much an individual wishes to behave consistently with what people important to him think. 


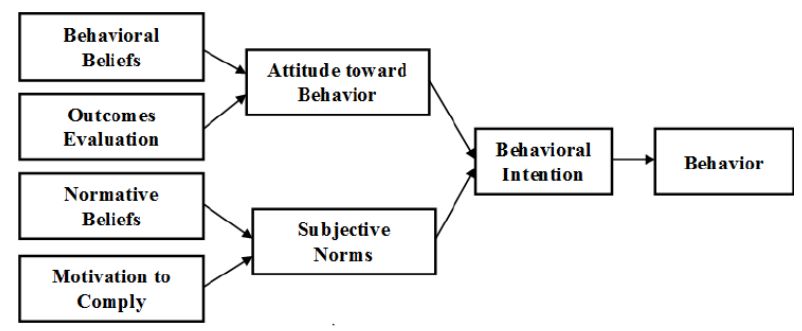

Figure 1 Theory of Reasoned Action

We expand TRA by including a personal moralityrelated variable of moral norm, which is different from subjective norm. TRA and its sibling TPB (theory of planned behavior) have been widely adopted to explain technology and non-technology's adoption behaviors. Nonetheless, they are often criticized by not including morality [7]. For example, Ajzen (1991)[2], who developed the TPB, recommended including moral norm as a behavioral intention predictor. Moral norms represent internalized and unconditional norms, values, and imperatives of important others. Although moral norms and subjective norms usually go together, they do not have to do so as they are different concepts. Manstead (2000)[35] has reviewed several studies indicating that moral norms can sometimes account for unique variance in behavioral intentions above and beyond that accounted for by attitudes and subjective norm. Several empirical studies also support the idea that the inclusion of moral norm could help increase TPB's explanatory power (e.g., [35] [42] [29]). Wan et al. (2014)[60] suggested that measuring moral norm from attitudes would provide a considerably precise conceptual distinctiveness. In the usage context of AI that may yield ethical issues, a person's own internalized moral norm is different from subjective norm that reflects others' expectations, and thus could be an important addition to increase explanatory power of AI use intention.

\section{Study Variables \& Research Model}

\subsection{Variables of Moral Psychology}

To examine how human's ethical beliefs ultimately affect the decision-making of AI adoption, we include key belief variables identified from the moral psychology discipline. According to moral psychology, a human is an independent and active learner, and can create and define environments rather than a passive being controlled by the environment or by subconscious and impulsive stimulus-response mechanism. That is, a person's ethics principles and judgements are formed through the dynamic interactions with those who can make ethical judgments and follow rules.

As for ethical decisions, there are two viewpoints in moral psychology. The principle of phenomenalism suggests that ethical decisions are grounded on rationality rather than emotion or desire. Moral psychology thus assumes that a person's ethical judgements and behaviors can be empirically explained through the understanding of complex psychological mechanisms. There is also an alternative view that much of the ethical/unethical decisions and behaviors are implicit or automatic, and thus individuals are unable to explicitly express the motivation of such behaviors [36]. Our research is grounded on the position that ethical decisions are driven by rationality rather than emotion or desire. Based on the positioning, we study if selfconsistency and social desirability, as key moral psychology variables, affect the attitudes toward AI in terms of its usefulness, subjective norm, and moral norm.

Self-consistency. According to self-concept theory, self-consistency represents the motivation to act in accordance with the self-concept and to maintain congruence between ideals and behaviors [12].

Social Desirability. As a concept that has been long studied in psychology as a cognitive variable, it refers to the fact that people often report or state inaccurately in order to be viewed favorably by others by presenting themselves in the best possible light in a social culture [14]. Social desirability, although a cognitive attribute [6], it is also related to an individual's emotional stability and consciousness [40].

\subsection{Attitudes/Beliefs toward AI}

We examine the bearing of the two moral psychology variables on a person's perception of AI's usefulness and his/her subjective and moral norms of $\mathrm{AI}$, and subsequently AI adoption intention.

Perceived Usefulness of AI. Usefulness perception is one of the most frequented indicators of user behaviors and naturally the relationship between usefulness perception of a technology and its adoption intention has been frequently examined. Thus, a research model is proposed to predict the intention to use the AI system through the lens of extended TRA and the chosen variables of moral psychology (Figure 1).

Subjective Norm for AI. As a core variable of TRA, subjective norm refers to "the perceived social pressure to perform or not to perform the behavior" in question [2] [3]. It is a person's normative beliefs about the extent 
to which other people who are important to the individual think they should or should not perform particular behaviors.

Moral Norm for AI. Moral norm is internalized and unconditional norms, values, and imperatives of important others. Thus, it is each person's own views about right and wrong as value judgmental, which have been learned during life [22] [41] [46]. It is different from utilitarian or hedonic attributes such as good/bad, beneficial/harmful, enjoyable/unenjoyable, nice/nasty, pleasant/unpleasant [22]. The moral norm as internalized values is manifested when a person understand the consequences of an action and willing to take responsibility for the consequences.

\subsection{Research Model}

There has been differing theoretical views on the dynamics among moral norm, attitudes, and behavioral intentions. Naturally empirical models depicted the relationship between moral norm and behavioral intentions differently depending on the study context. In certain research moral norm substituted attitudes [13] and, in others, moral norm bypasses attitudes, directly affecting behavioral intentions [15]. In our study, we posit that moral norm for AI directly influences adoption intentions independent of other variables.

The research model that incorporates the study variables are presented in Figure 1.

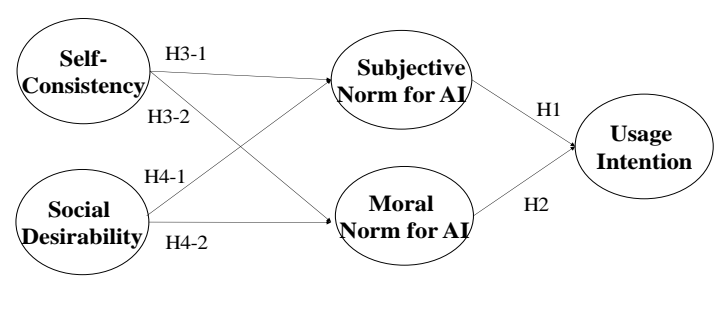

$<$ Figure 1 > Research Model

\section{Hypotheses}

\subsection{Subjective Norm for AI \& AI Adoption Intention}

Subjective norm of AI refers to a "person's perception that most people who are important to him/her think that he/she should or should not perform the behavior in question [3]". That is, subjective norm is judgment of most people to approve or disapprove on a particular behavior, which translates into perceived social pressure to perform or not to perform it [2]. AI is a type of information technology and we anticipate that the dynamics between behavioral intention and subjective norm of TRA hold consistent in the AI context. Thus, the following hypothesis is proposed:

H1: A person's subjective norm for AI is positively associated with his/her intention to adopt it.

\subsection{Moral Norm for AI \& AI Adoption Intention}

Moral norm for AI refers to individual beliefs about what is right and wrong [41] or internalized norms and values of important others that have been learned during life [46]. According to Norm-Activation theory [46], a person's moral behavior is his/her manifestation of the personal norm to act in a certain way. When an individual knows the consequences of his/her actions and willing to take responsibility for them, his/her moral norm is activated. When there is social dilemma, moral norm is known to affect personal behaviors [59].

Several empirical studies support that including moral norm could increase the explanatory power of a person's behavior (e.g., [35] [42] [29]). In the medical field, it was revealed that moral norm had a stronger influence on people's adoption intention of the AIDS vaccine than other study variables [30]. Moral norm was incorporated into TPB to predict recycling intention [25] [54]. Harland et al. (1999)[25] proved that, in different contexts, moral norm significantly increases variance in explaining behavioral intentions. Wan et al. (2014)[60] suggested that measuring moral norm would provide a conceptual distinctiveness. These studies demonstrate importance of moral norm as a concept distinct from subjective norm.

In the AI context that may carry ethical implications, moral norm for AI is expected to be a meaningful addition to better explain user adoption intentions. In fact, adopting an AI system by an individual demands its evaluation through ethical lens [8] and thus the inclusion of moral norm is well warranted. That is, the stronger a person's moral norm for AI, she/he may apply a stricter internalized principle toward AI-related decisions. In particular, since the context of our study is the 'user's attitude on a specific AI product after recognizing that $\mathrm{AI}$ in general may have ethical dilemma', moral norm in our model is negatively associated with intention to adopt. With that, the following hypothesis is proposed:

H2: A person's moral norm for AI is negatively associated with his/her intention to adopt it.

\subsection{Effects of Self-Consistency}


Self-consistency is a person's desire to behave consistent with his/her ideals [12]. When a person of high self-consistency feels strong about acting in accordance with the self-concept and ideals, she/he may have a high ethical identity and a strong desire to behave according to his/her own ethical values. With a higher ethical identity, a person views his/her life more through the ethical lens, pursues a life that accords with his/her ethical ideals, and feels stronger responsibilities to act on ethical judgements [12]. With the strong sense of obligations, she/he may become more conscious of how others perceive a particular subject and perceive more social pressures to perform or not to perform the behavior approved or disapproved by most people. This conformance between an individual's self-consistency and social pressure is expected strong as a person's judgement of ethics is shaped within the community context. The adoption and usage of AI may not be an exception. Thus the high association between the level of self-consistency and subjective norm in the AI context is hypothesized:

H3-1. A person's self-consistency is positively associated with his/her subjective norm for AI.

As stated, those with high moral identity is expected to have strong tendency and desire to be consistent in moral judgements and behaviors, and thus is expected to reveal little discrepancy between them. Meanwhile, moral norm for AI refers to personalized and internalized beliefs about what is right and wrong about AI. When a person is more inclined to or strive for maintaining moral consistency, it is natural to expect that she/he is going to develop a stronger internalized attitude or moral norm on certain subjects including AI and adhere to it. Self-congruity theory also sheds insights into their coherence relationship. According to the theory, consumers prefer a brand which has images congruent with their self-concept. Actual self-congruity effects stem from a self-consistency motive which involves the tendency to behave in a way to protect an individual's present self-image. In other words, selfcongruity effects arise from self-consistency [1]. The degree of congruence between self-concept and a typical brand image enhances the brand evaluation by the user [48] [23]. If the logic is extended to the relationship between self-consistency and moral norm of AI, it is expected that a person's tendency to be congruent between judgement and behaviors is expected to influence the shaping of his/her internal moral norms on AI. Thus, it is proposed that:

H3-2. A person's self-consistency is positively associated with his/her moral norm for AI.

\subsection{Effects of Social Desirability}

Social desirability is defined as an individual tendency to conduct what is considered socially desirable or correct within a cultural context [14]. A person's social desirability is affected by his/her personality such as emotional stability and consciousness [40] and age [13]. Oftentimes, social desirability biases a person's selfreport response for it to manifest more ethical to others [11]. Due to the bias, a person with higher sense of social desirability might accept what's happening throughout the society more positively.

As stated, social desirability of a person tends to align his/her views with those hold by the majority in social issues. For example, research reveals a significant effect of social desirability in people's support for a woman president in US [49]. Meanwhile, the subjective norm represents the perceived social pressure for actions felt by an individual from important others [2] [3]. Naturally, a person with a strong sense of social desirability is expected to psychologically become more sensitive about how important others think about AI and may develop similar views. In fact, the connection between social desirability and subjective norm has been empirically examined in other subject areas. We anticipate such relationship in the context of AI. Therefore, the following hypothesis is proposed.

H4-1. A person's social desirability is positively associated with his/her subjective norm for AI.

Moral norm for AI refers to individual beliefs and values about what is right and wrong or internalized norms [41]. A person with strong sense to think and behave socially desirable is expected to develop a strong sense of moral norm on AI aligned with others' views. In other words, the more a person is keen about ethical behaviors commonly expected by the society, the more he/she may become sensitive about ethical issues raised by the community in using AI. These issues include ethics of responsibility, principles and behaviors in using AI. It is therefore proposed that:

H4-2. A person's social desirability is positively associated with his/her moral norm for AI.

\section{Research Method}

We used structural equation modeling using survey data to empirically test the hypotheses. For survey distribution, a sample was drawn based on the stratified sampling technique to evenly draw respondents across regions, ages, and gender from the panel of a survey institute. The survey solicitation was emailed to the 
respondents, which included an online link to the survey. To encourage their active participation, each respondent was paid about $\$ 5$ after completing the survey. The survey limited data collection from participants of at least 20 years old and the online survey began with the question for the filtering. The justification was that the morality scenarios to be presented as part of the survey require for the respondent to able to drive and to afford product purchase.

492 survey responses returned and, through their manual reviews, 440 responses are used for analysis after dropping 52 responses that were clearly lacking their response reliability (e.g., all answers were identical). 24 survey questions that represent 5 variables were initially derived from existing studies, but survey data related to 19 questions were used after their reliability testing based on exploratory factor analysis. All responses were based on the 7-point Likert scale. SPSS 23.0 was used to obtain descriptive statistics and to conduct exploratory factor analysis and smartPLS 2.0 was the platform for SEM estimation. The respondents' demographics are summarized in Table 1.

\section{Results}

\subsection{Profile of Survey Participants}

Table 1 summarizes the demographics of the surveyed.

$<$ Table 1> Summary of the demographics of the surveyed

\begin{tabular}{|c|c|c|}
\hline \multicolumn{2}{|c|}{ Demographics } & $\begin{array}{c}\text { Total } \\
(n=440)\end{array}$ \\
\hline \multirow{4}{*}{ Age } & 20 ’s & $108(24.5)$ \\
\hline & 30 's & $104(23.6)$ \\
\hline & 40 's & $118(26.8)$ \\
\hline & 50 's or older & $110(25.1)$ \\
\hline \multirow{2}{*}{ Gender } & Male & $207(47.0)$ \\
\hline & Female & $233(53.0)$ \\
\hline \multirow{4}{*}{ Education } & $\begin{array}{l}\text { High school } \\
\text { diploma }\end{array}$ & $76(17.3)$ \\
\hline & $\begin{array}{l}\text { Studying for } \\
\text { undergraduate }\end{array}$ & $33(7.5)$ \\
\hline & $\begin{array}{l}\text { Graduated with } \\
\text { bachelor degree }\end{array}$ & $286(65.0)$ \\
\hline & $\begin{array}{l}\text { Studying for or } \\
\text { graduated with } \\
\text { master degree }\end{array}$ & $45(10.2)$ \\
\hline \multirow{2}{*}{ Occupation } & Student & $36(8.2)$ \\
\hline & firm employee & $258(58.6)$ \\
\hline
\end{tabular}

\begin{tabular}{|l|c|c|}
\hline & homemaker & $61(13.9)$ \\
\cline { 2 - 3 } & professional & $39(8.9)$ \\
\cline { 2 - 3 } & others & $46(10.5)$ \\
\cline { 2 - 3 } & Not used AI & $132(30.0)$ \\
\hline
\end{tabular}

\subsection{Measurement}

The factor analysis revealed that commonality exceeded 0.724, and factor loadings revealed 6 factors, with no multiple loading items for only one factor of 0.6 or more. The results of the exploratory factor analysis revealed that the KMO (Kaiser-Meyer-Olkin) value for the sample was 0.856 , which confirms that the data set is valid for factor analysis. In addition, the sphere formation test value for the sample was $\mathrm{x}^{2}=5674.294$ $(\mathrm{df}=171, \mathrm{p}<.001)$, and the cumulative total variance of the factors was $75.4 \%$, which is judged to be suitable for the factor analysis. Reliability of the eight identified factors was confirmed by Cronbach's $\alpha$ coefficient, which was higher than 0.860 (except Social Desirability 0.596) displaying high credibility. Table 2 summarizes the results of the exploratory factor and reliability analyses.

$<$ Table 2> Exploratory factor analysis $(n=440)$

\begin{tabular}{|c|c|c|c|c|}
\hline Factors & Items & $\begin{array}{c}\text { Loadin } \\
\mathrm{g}\end{array}$ & $\begin{array}{l}\text { Composite } \\
\text { Reliability }\end{array}$ & $\begin{array}{c}\text { Cronba } \\
\text { chAlph } \\
\mathrm{a}\end{array}$ \\
\hline \multirow{5}{*}{$\begin{array}{l}\text { Usage } \\
\text { Intention } \\
\text { (UI) }\end{array}$} & UI1 & 0.874 & \multirow{5}{*}{0.959} & \multirow{5}{*}{.946} \\
\hline & UI2 & 0.869 & & \\
\hline & UI3 & 0.868 & & \\
\hline & UI4 & 0.865 & & \\
\hline & UI5 & 0.850 & & \\
\hline \multirow{4}{*}{$\begin{array}{l}\text { Subjective } \\
\text { Norm } \\
\text { (SN) }\end{array}$} & SN1 & 0.859 & \multirow{4}{*}{0.948} & \multirow{4}{*}{.927} \\
\hline & SN2 & 0.840 & & \\
\hline & SN3 & 0.836 & & \\
\hline & SN4 & 0.820 & & \\
\hline \multirow{4}{*}{$\begin{array}{l}\text { Moral } \\
\text { Norm } \\
(\mathrm{MN})\end{array}$} & MN1 & 0.891 & \multirow{4}{*}{0.905} & \multirow{4}{*}{.860} \\
\hline & MN2 & 0.880 & & \\
\hline & MN3 & 0.832 & & \\
\hline & MN 4 & 0.715 & & \\
\hline \multirow{3}{*}{$\begin{array}{c}\text { Self- } \\
\text { Consist. } \\
(\mathrm{SC})\end{array}$} & SCI1 (r) & 0.896 & \multirow{3}{*}{0.910} & \multirow{3}{*}{.860} \\
\hline & $\mathrm{SC} 2(\mathrm{r})$ & 0.885 & & \\
\hline & SC3 (r) & 0.844 & & \\
\hline \multirow{3}{*}{$\begin{array}{c}\text { Social } \\
\text { Desirability } \\
\text { (SD) }\end{array}$} & SD1 & 0.751 & \multirow{3}{*}{0.789} & \multirow{3}{*}{.596} \\
\hline & SD2 & 0.730 & & \\
\hline & SD3 & 0.688 & & \\
\hline
\end{tabular}

Note 2: KMO(Kaiser-Meyer-Olkin Measure of Sample Adequacy) $=0.856$, Total Variance explained $=75.4 \%$, Bartlett's Test of Sphericity $=5674.294(\mathrm{df}=171$, Sig. $=0.000)$ 


\subsection{Measurement Validation}

The validity and appropriateness of the measurement model were determined before testing the hypotheses of this study. First, as shown in Table 3, the AVE (average variance extracted) exceeds 0.555, which indicates convergent validity [9]. Composite reliability $(\mathrm{CR})$, which is an index that measures the feasibility of the measurement model, exceeds 0.789 , indicating reliability. Also, Cronbach's alpha measures the reliability of each factor for which 0.6 is considered a threshold value [37]. Except for Social Desirability (0.596), all other Cronbach's alphas are higher than 0.860 indicating reliability in the factor structure. Although the Cronbach's alpha for Social Desirability is relatively lower than the others, it surpassed the generally accepted threshold value [37]. The fit/quality of a structural model needs to have a positive redundancy [53]. In our study, all redundancies except moral norm are positive indicating model fit. The negative redundancy of moral norm also indicates its fit as its paths has been rejected [53]. In this study, the size of goodness-of-fit in the PLS path model is regarded as large if the value is 0.36 or larger; and medium if the value is $0.25 \sim 0.36$, the value for moral TRA was 0.284 thus showing medium goodness-of-fit [53].

$<$ Table 3> Correlations and Average Variance Extracted

\begin{tabular}{|l|c|c|c|c|c|}
\hline \multicolumn{1}{|c|}{ Var. } & $(1)$ & $(2)$ & $(3)$ & (4) & (5) \\
\hline (1) SC & 0.88 & & & & \\
\hline (2) SD & $.21^{* *}$ & 0.74 & & & \\
\hline (3) SN & $-.12^{*}$ & $.15^{* *}$ & 0.91 & & \\
\hline (4) $\mathrm{MN}$ & -0.04 & $.26^{* *}$ & -0.01 & 0.84 & \\
\hline (5) UI & 0.01 & $.13^{* *}$ & $.62^{* *}$ & $-.12^{*}$ & 0.91 \\
\hline
\end{tabular}

Note 1: The diagonal values are the square root of AVE Note $2: * \mathrm{p}<0.05, * * \mathrm{p}<0.01$

\subsection{Results}

The hypotheses in this study were tested through path counting and the valence of each path coefficient was confirmed by setting 5,000 bootstrapping specimens [24]. The significance of individual paths is summarized in Table 4 five out of 7 paths exhibited a pvalue less than 0.05. All hypotheses except $\mathrm{H} 3-2$ have been accepted. The explanatory power of the research model is also shown. The adjusted R-squared value shows that the constructs in the model together accounted for $39.7 \%$ of Intention to use in moral AI.

$<$ Table 4> Path Coefficients and hypotheses testing

\begin{tabular}{|c|c|c|c|c|}
\hline Hyp & $\begin{array}{c}\text { Sample } \\
\text { Mean }\end{array}$ & $\begin{array}{c}\text { Standard } \\
\text { Deviation }\end{array}$ & T Stat & Result \\
\hline H1 & 0.621 & 0.036 & 17.131 & Accept \\
\hline H2 & -0.112 & 0.052 & 2.142 & Accept \\
\hline H3-1 & 0.170 & 0.057 & 2.932 & Accept \\
\hline H3-2 & 0.102 & 0.057 & 1.788 & Reject \\
\hline H4-1 & 0.185 & 0.050 & 3.660 & Accept \\
\hline H4-2 & 0.295 & 0.047 & 6.147 & Accept \\
\hline
\end{tabular}

Note: ${ }^{*} \mathrm{p}<0.05(\mathrm{t}>1.96),{ }^{* *} \mathrm{p}<0.01(\mathrm{t}>2.58),{ }^{* *} \mathrm{p}<0.001(\mathrm{t}>3.30)$

\section{Discussions}

The empirical study clearly shows the significance of moral/ethical variables in directly and indirectly affect the usage intention of AI products/services. First, subjective norm is significantly associated with intention to use of AI (H1), which is consistent with other TRA-based studies that confirms the role of subjective norm in new IT adoption (e.g., [2] [55]). This tells the effectiveness of the marketing plan factor in the prediction of the psychology of AI products/services of figures considered important by a target customer. This might help overcome possible ethical concerns a customer might have.

Moral norm's negative influence on the adoption intention of AI products is confirmed (H2 supported). It is consistent with other empirical findings [13] that moral norm is an influential force in user behaviors in IT adoption and usage. Theoretically, this confirms that the traditional elements of TRA (i.e., perceived usefulness, subjective norm) are not enough to explain user rejection of AI when it has ethical issues. This clearly sends a practical message to businesses that people's ethical dilemma has a bearing on their usage of AI products/services and, to be successful, AI service/product providers should find ways (e.g., communication strategy) to relieve or remove the negative perceptions that may raise to the potential adopters. For example, if possible, businesses may carefully evaluate what AI functions can trigger the conflict with a person's moral norm and those that can cause such concerns may be set aside or even entirely removed from the product/service offering to change user perceptions.

Self-consistency is significantly affects a person's subjective norm of AI (H3-1), but not with moral norm (H3-2). Self-consistency motivates a person to maintain consistency between ideals and behaviors [12]. It is therefore highly internalized and salient self-concept that promotes moral behaviors. The empirical results 
indicate that such subjective assessment of moral behaviors projects to the lens through which an individual judges the opinions of others important to him/her. This also implies that the understanding of an individual on important others' opinions and views can be biased by subjective moral standards and values. But such self-consistency fails to influence the shaping and internalize moral norm for the individual. Remember that the moral norm of this study is about internalized views of AI's morality. That is, the general selfconsistency principle of an individual has little bearing on shaping the morality attitude of AI services/products.

Values for social desirability is significantly associated with subjective norm of AI (H4-1) and moral norm of AI (H4-2). Especially the effect of social desirability on moral norm of AI provides an important theoretical links that explains how moral psycholology can lead to AI's usage intention via changing and reshaping the TRA's core attitude morality variables. As for the link between social desirability on moral norm, a person's basic ethics is naturally formed social desirability that may be largely a consequence of public and private education and pervasive culture of managed or naturally formed within a society. That is, ethical issues of AI emanating from social desirability is ultimately affect AI usage intention through a person's moral norm as an attitude variable. With this, it is important that a society's education system and shaping of culture through regulations and policies that can resolve ethical dilemmas could ultimately have a significant bearing AI's usage intention of people. Further, the influence of social desirability on subjective norm and perceived usefulness further galvanize the significant effects that the moral psychology variable ultimately has on AI's adoption. A caution, however, is necessary that our research used a self-reported perceptions of social desirability, which could be different from actual behaviors or from others' assessment.

Other than morality, the economics of time and the psychology of space are also important for the usage intention of new digital technologies such as smart home [26]. Nevertheless, morality has significant impact on intention to use new digital products in ways quite contrary to the expectations of product designers [26].

As Macintyre (1981, 1998)[33] [34] argued, designers may not even recognize the seriousness of the morality issues in AI product design. Based on relativism, in particular, what is moral and what is not are very personal and hard to be uniformly decided. This means that user's psychological mechanism should be carefully investigated to make the AI products socially and economically successful. The practitioners and designers of an AI product must consider the moral priorities for their potential customers. Hence, it is useful top conduct empirical studies of new AI products that explore the paradigms of the moral order can, and do take place.

\section{Conclusion}

AI has been rising rapidly but it also engenders serious ethical issues such as accountability. Several studies attempted to explain AI adoption through the traditional theories of technology adoption. In the wake of many different ethical issues it raises, however, the traditional factors of decision making may not be enough to fully explicate a person's adoption intentions of AI. This is because the traditional theories largely view technology adoption through the utilitarian or hedonic lenses. To examine potential effects the ethical side of AI might have on prospective users, this study explores the role of variables related to ethics. For this, we expanded Theory of Reasoned Action (TRA) by adding variables that measure a person's morality, which includes moral norm, social desirability and selfconsistency. The empirical results indicate a direct and indirect role of the included variables in explaining users' AI adoption intentions. The distinctive patterns emerged highlight the theoretical and practical importance of embracing ethical factors to better explain AI adoption behaviors among prospective users.

\section{References}

[1] Aguirre-Rodriguez, A., Bosnjak, M., and Sirgy, M. J. (2012), Moderators of the self-congruity effect on consumer decision-making: A meta-analysis, Journal of Business Research, 65(8), 1179-1188.

[2] Ajzen, I. (1991). The theory of planned behavior. Organizational Behavior and Human Decision Processes, 50, 179-211.

[3] Ajzen, I., \& Driver, B. L. (1991). Prediction of leisure participation from behavioral, normative, and control beliefs: An application of the theory of planned behavior. Leisure Sciences, 13, 185-204.

[4] Ajzen, I., \& Fishbein, M. (1975). A Bayesian analysis of attribution processes. Psychological Bulletin, 82(2), 261.

[5] Akinnuwesi, B. A., Uzoka, F. M. E., Okwundu, O. S., \& Fashoto, G. (2016). Exploring biometric technology adoption in a developing country context using the modified UTAUT. International Journal of Business Information Systems, 23(4), 482-521.

[6] Andrejevic, M. (2017). Digital Citizenship and Surveillance| To Pre-Empt A Thief. International Journal of Communication, 11, 18.

[7] Armitage, C. J., \& Conner, M. (2001). Efficacy of the theory of planned behaviour: A meta-analytic review. British Journal of Social Psychology. 40(4), 471-499.

[8] Arnold, T., \& Scheutz, M. (2018). The "big red button" 
is too late: an alternative model for the ethical evaluation of AI systems. Ethics and Information Technology, 20(1), 59-69.

[9] Bagozzi, R. P., \& Yi, Y. (1988). On the evaluation of structural equation models. Journal of the Academy of Marketing Science, 16(1), 74-94.

[10] Bandura, A. (1986). The explanatory and predictive scope of self-efficacy theory. Journal of Social and Clinical Psychology, 4(3), 359-373.

[11] Bay, D., \& Nikitkov, A. (2011). Subjective probability assessments of the incidence of unethical behavior: the importance of scenario-respondent fit. Business Ethics: A European Review, 20(1), 1-11.

[12] Blasi, A. (1983). Moral cognition and moral action: A theoretical perspective. Developmental review, 3(2), 178-210.

[13] Chan, L., \& Bishop, B. (2013). A moral basis for recycling: Extending the theory of planned behaviour. Journal of Environmental Psychology, 36, 96-102.

[14] Crowne, D. P., \& Marlowe, D. (1960). A new scale of social desirability independent of psychopathology. Journal of consulting psychology, 24(4), 349.

[15] Botetzagias, I., Dima, A. F., \& Malesios, C. (2015). Extending the theory of planned behavior in the context of recycling: The role of moral norms and of demographic predictors. Resources, Conservation and Recycling, 95, 58-67.

[16] Choi, J., \& Kim, S. (2016). Is the smartwatch an IT product or a fashion product? A study on factors affecting the intention to use smartwatches. Computers in Human Behavior, 63, 777-786.

[17] Conitzer, V., Sinnott-Armstrong, W., Borg, J. S., Deng, Y., \& Kramer, M. (2017). Moral Decision Making Frameworks for Artificial Intelligence. In AAAI (pp. 4831-4835).

[18] Davis, F. D. (1989). Perceived usefulness, perceived ease of use, and user acceptance of information technology. MIS quarterly, 319-340.

[19] Davis, F. D., Bagozzi, R. P., \& Warshaw, P. R. (1989). User acceptance of computer technology: a comparison of two theoretical models. Management Science, 35(8), 982-1003.

[20] Davis, F. D., Bagozzi, R. P., \& Warshaw, P. R. (1992). Extrinsic and intrinsic motivation to use computers in the workplace 1. Journal of Applied Social Psychology, 22(14), 1111-1132.

[21] Fan, W., Liu, J., Zhu, S., \& Pardalos, P. M. (2018). Investigating the impacting factors for the healthcare professionals to adopt artificial intelligence-based medical diagnosis support system (AIMDSS). Annals of Operations Research, 1-26.

[22] Godin, G., Conner, M., \& Sheeran, P. (2005). Bridging the intention-behaviour gap: The role of moral norm. British Journal of Social Psychology, 44(4), 497-512.

[23] Graeff, T. R. (1996), Image congruence effects on product evaluations: The role of self-monitoring and public/private consumption, Psychology and Marketing, 13(5), 481-499.

[24] Hair, J. F., Ringle, C. M., \& Sarstedt, M. (2011). PLS-SEM: Indeed a silver bullet. Journal of Marketing Theory and Practice, 19(2), 139-152.
[25] Harland, P., Staats, H., \& Wilke, H. A. (1999). Explaining proenvironmental intention and behavior by personal norms and the Theory of Planned Behavior 1. Journal of Applied Social Psychology, 29(12), 2505-2528.

[26] Harper, R. (Ed.). (2006). Inside the Smart Home. Springer Science \& Business Media.

[27] Hlee, S., Lee, J., Moon, D., \& Yoo, C. (2017). The acceptance of 'intelligent trade shows': Visitors' evaluations of IS innovation. Information Systems Frontiers, 19(4), 717-729.

[28] Hongladarom, S. (2017). Information Technology: Ethics. Encyclopedia of Global Bioethics, 1-8.

[29] Huijts, N. M., Molin, E. J., \& van Wee, B. (2014). Hydrogen fuel station acceptance: a structural equation model based on the technology acceptance framework. Journal of Environmental Psychology, 38, 153-166.

[30] Juraskova, I., O'Brien, M., Mullan, B., Bari, R., Laidsaar-Powell, R., \& McCaffery, K. (2012). HPV vaccination and the effect of information framing on intentions and behaviour: An application of the theory of planned behaviour and moral norm. International Journal of Behavioral Medicine, 19(4), 518-525.

[31] Katz, E. (1959).Mass communications research and the study of popular culture: An editorial note on a possible future for this journal, Public Communication, 2, 1-6

[32] Kolbjørnsrud, V., Amico, R., \& Thomas, R. J. (2016). How artificial intelligence will redefine management. Harvard Business Review, 2, 1-6.

[33] Macintyre, A (1981) After Virtue, London: Duckworth.

[34] Macintyre, A (1998) A Short History of Ethics, London: Routledge (2nd edn).

[35] Manstead, A. S. R. (2000). The Role of Moral Norm in the Attitude-behavior Relation. In D. J. Terry \& M. A. Hogg (Eds.), Attitudes, behaviour and social context: The role of norms and group membership (pp. 11-30). Mahwah, NJ: Lawrence Erlbaum Associates.

[36] Narvaez, D., \& Lapsley, D. K. (2005). The psychological foundations of everyday morality and moral expertise. Character Psychology and Character Education, 140-165.

[37] Nunnally, J. (1978) Psychometric Method. McGraw-Hill, New York.

[38] O'Leary, D. E. (1998). Enterprise knowledge management. Computer, 31(3), 54-61.

[39] O'Leary, D. E. (2013). Artificial intelligence and big data. IEEE Intelligent Systems, 28(2), 96-99.

[40] Ones, D. S., Viswesvaran, C., \& Reiss, A. D. (1996). Role of social desirability in personality testing for personnel selection: The red herring. Journal of Applied Psychology, 81(6), 660.

[41] Parker, D., Manstead, A. S. R., \& Stradling, S. G. (1995). Extending the theory of planned behaviour: The role of personal norm. British Journal of Social Psychology 34, 127-137.

[42] Rivis, A., Sheeran, P., \& Armitage, C. J. (2009). Expanding the affective and normative components of the theory of planned behavior: A meta-analysis of anticipated affect and moral norms. Journal of Applied Social Psychology, 39(12), 2985-3019.

[43] Rogers, E. M. (1995). Diffusion of Innovations: Modifications of a Model for Telecommunications. In Die diffusion von innovationen in der 
telekommunikation (pp. 25-38). Springer, Berlin, Heidelberg.

[44] Ruggiero, T. E. (2000). Uses and gratifications theory in the 21st century. Mass Communication \& Society, 3(1), 3-37.

[45] Rubin, A. M. 1993. Audience activity and media use. Communication Monographs, 60: 98-105.

[46] Schwartz, S. H. (1977). Normative influences on altruism1. Advances in Experimental Social Psychology (Vol. 10, pp. 221-279). Academic Press.

[47] Shoemaker, P. J., \& Tetlock, P. E. (2017). Building a more intelligent enterprise. MIT Sloan Management Review, 58(3), 28.

[48] Sirgy, M. J., Johar, J. S., Samli, A. C., and Claiborne, C. B. (1991), Self-congruity versus functional congruity: Predictors of consumer behavior, Journal of the Academy of Marketing Science, 19(4), 363-375.

[49] Streb, C., Ritchie, C., Long, D. L., Kögerler, P., \& Cronin, L. (2007). Modular Assembly of a Functional Polyoxometalate-Based Open Framework Constructed from Unsupported AgI $\cdots$ AgI Interactions. Angewandte Chemie International Edition, 46(40), 7579-7582.

[50] Sundar, S. S., \& Limperos, A. M. (2013). Uses and grats 2.0: New gratifications for new media. Journal of Broadcasting \& Electronic Media, 57(4), 504-525.

[51] Klapper, J. T. (1963).Mass communication research: An old road resurveyed, The Public Opinion Quarterly, 27(4), 515-527.

[52] Taylor, S., \& Todd, P. (1995). Decomposition and crossover effects in the theory of planned behavior: A study of consumer adoption intentions. International Journal of Research in Marketing, 12(2), 137-155.

[53] Tenenhaus, M., Vinzi, V. E., Chatelin, Y. M., \& Lauro, C. (2005). PLS path modeling. Computational Statistics \& Data Analysis, 48(1), 159-205.

[54] Tonglet, M., Phillips, P. S., \& Read, A. D. (2004). Using the Theory of Planned Behaviour to investigate the determinants of recycling behaviour: a case study from
Brixworth, UK. Resources, Conservation and Recycling, 41(3), 191-214.

[55] Vallerand, R. J., \& Blssonnette, R. (1992). Intrinsic, extrinsic, and amotivational styles as predictors of behavior: A prospective study. Journal of Personality, 60(3), 599-620.

[56] Van der Linden, S. (2011). Charitable intent: A moral or social construct? A revised theory of planned behavior model. Current Psychology, 30(4), 355-374.

[57] Venkatesh, V., \& Davis, F. D. (1996). A model of the antecedents of perceived ease of use: Development and test. Decision Sciences, 27(3), 451-481.

[58] Venkatesh, V., Morris, M. G., Davis, G. B., \& Davis, F. D. (2003). User acceptance of information technology: Toward a unified view. MIS quarterly, 425-478.

[59] Von Borgstede, C., Dahlstrand, U., \& Biel, A. (1999). From Ought to is: Moral Norms in Large-scale Social Dilemmas. Goteborg Psychological Reports, 29.

[60] Wan, C., Shen, G. Q., \& Yu, A. (2014). The moderating effect of perceived policy effectiveness on recycling intention. Journal of Environmental Psychology, 37, 5560.

[61] Wenger, E. (2014). Artificial intelligence and tutoring systems: computational and cognitive approaches to the communication of knowledge. Morgan Kaufmann.

[62] Wolkenstein, A. (2018). What has the Trolley Dilemma ever done for us (and what will it do in the future)? On some recent debates about the ethics of self-driving cars. Ethics and Information Technology, 1-11.

[63] Yang, H., \& Lee, H. (2018). Understanding user behavior of virtual personal assistant devices. Information Systems and e-Business Management, 1-23.

[64] Zhang, J., \& Curley, S. P. (2018). Exploring Explanation Effects on Consumers' Trust in Online Recommender Agents. International Journal of Human-Computer Interaction, 34(5), 421-432. 